Revue d'histoire de l'Amérique française

RAS REVUE D.HISTOIRE DE L'AMÉRIQUE FRANÇAISE

\title{
Un nouveau manuscrit des lettres de Marie de l'Incarnation
}

Dom Guy Oury

Volume 21, numéro 1, juin 1967

URI : https://id.erudit.org/iderudit/302644ar

DOI : https://doi.org/10.7202/302644ar

Aller au sommaire du numéro

Éditeur(s)

Institut d'histoire de l'Amérique française

ISSN

0035-2357 (imprimé)

1492-1383 (numérique)

Découvrir la revue

Citer cet article

Oury, D. G. (1967). Un nouveau manuscrit des lettres de Marie de l'Incarnation. Revue d'histoire de l'Amérique française, 21(1), 51-63.

https://doi.org/10.7202/302644ar d'utilisation que vous pouvez consulter en ligne.

https://apropos.erudit.org/fr/usagers/politique-dutilisation/ 


\section{UN NOUVEAU MANUSCRIT DES LETTRES DE MARIE DE L'INCARNATION *}

Au nombre des acquisitions récentes faites par les Archives publiques du Canada, à Ottawa, l'une des plus précieuses serait un manuscrit intitulé: "Lettres morales et édifiantes de Marie de l'Incarnation, Supérieure des Ursulines au Canada (Amérique)". Le vendeur, le Goodspedd's Book Shop de Boston (18 Beacon Street, Boston 8, Mass.), présentait le manuscrit comme une transcription du $\mathrm{XVII}^{\mathrm{e}}$ siècle, contenant 183 lettres de l'Ursuline (119 des 132 Lettres spirituelles éditées en 1681 par Dom Claude Martin, et 64 des 89 lettres historiques) ; il rappelait à cette occasion que les lettres avaient fait l'objet d'éditions en 1677 (édition partielle dans la Vie de la Vénérable Mère Marie de l'Incarnation, première supérieure des Ursulines de la Nouvelle-France, tirée de ses Lettres et des Écrits ${ }^{1}$, qui contient en effet de nombreux extraits de lettres), en $1681^{2}$, en $1857^{3}$, et en

* Les nombreuses notes réunies sur le manuscrit d'Ottawa par une Moniale de l'Abbaye Sainte-Marie-des-Deux-Montagnes nous ont été extrêmement précieuses pour la rédaction de cet article; nous sommes heureux de la remercier ici d'avoir bien voulu nous permettre de les utiliser.

1 Par Dom Claude Martin, religieux bénédictin de la Congrégation de Saint-Maur (à Paris, chez Louis Billaine).

2 Chez Louis Billaine également). Le fonds de commerce de Louis Billaine ayant été cédé peu après à deux "libraires", Martin Jouvenel et Antoine Vuarin, il existe actuellement plusieurs exemplaires où le nom de Louis Billaine a été masqué par un papillon portant une nouvelle adresse et une date nouvelle; on possède ainsi au moins trois éditions fictives:

a) l'une de 1684, chez Vuarin (il en existe un exemplaire à la Bibliothèque municipale du Mans) ;

b) la seconde de 1686, chez Jouvenel (il en existe un exemplaire à la Bibliothèque municipale de Montréal);

c) en 1696, Antoine Vuarin fit réimprimer la page de titre selon un libellé quelque peu différent: "Lettres spirituelles et historiques de la Vénérable Mère...", mais la p. 675 portant le privilège royal et l'achevé d'imprimer est restée inchangée (la Bibliothèque municipale de Montréal vient d'en acquérir un exemplaire). La même année, Vuarin refit la page de titre de la Vie, parue chez Louis Billaine en 1677. La Bibliothèque Nationale de Paris possède les deux "éditions" de la Vie.

3 Edition anonyme (chez F. Thibaud à Clermont-Ferrand), 388 pp.; c'est un choix de lettres. 
$1876^{4}$; il signalait enfin que le manuscrit retrouvé par lui contenait de nombreuses variantes textuelles et que la distribution des Lettres différait quelque peu de l'édition de Dom Claude Martin.

Le manuscrit acquis par les Archives d'Ottawa est un recueil de 17 cahiers in-quarto, contenant en tout 415 pages 5. Il ne donne aucune lettre qui ne se trouve déjà dans la collection de Dom Claude Martin. Voici une table indiquant le contenu du manuscrit et sa numérotation propre en regard de celle de Dom Claude Martin :

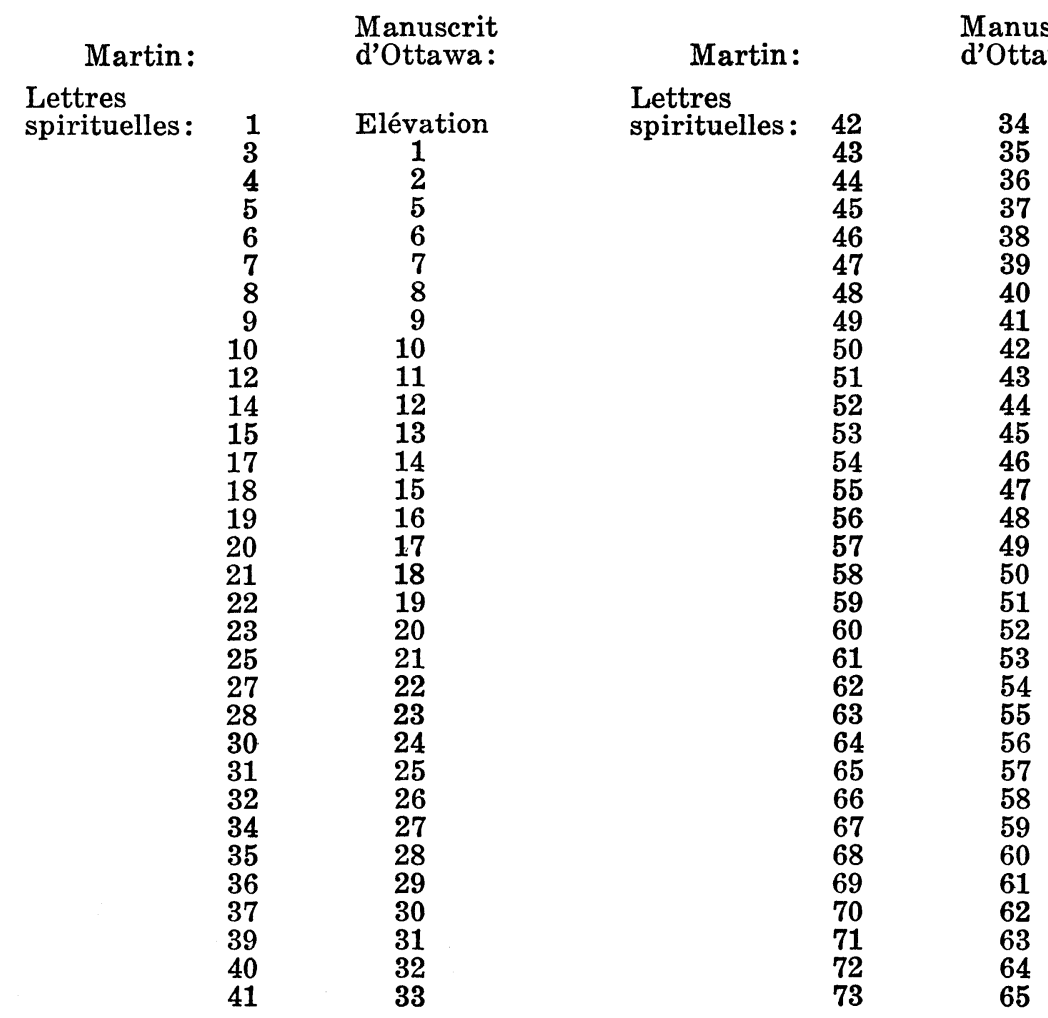

${ }^{4}$ Lettres de la Révérende Mère Marie de l'Incarnation, par l'abbé Richaudeau (2 vol., Casterman, Tournai), XIX-557, 560 pp.

5 La pagination du copiste cesse à la page 174 . 
Martin :

Lettres

spirituelles:
Martin :

Lettres

spirituelles : 129

131

$132 \quad 119$

Lettres

historiques :

1
Manuscrit d'Ottawa :

117

118

119

71

71

73

74

75

76

77

78

79

80

81

82

83

84

85

86

87

88

89

90

91

92

93

93 (sic)

104

105

106

107

108

109

110

111

112

113

114

116

117

118

119

120

121

122

123

124

125

126

127

128

95

96

97

98

99

100

101

102

103

104

105

106

107

108

109

110

111

112

113

114

114 (sic)

115

1
2
3
4
6
7
8
9
11




\begin{tabular}{|c|c|c|c|c|c|}
\hline \multicolumn{2}{|l|}{ Martin: } & $\begin{array}{l}\text { Manuscrit } \\
\text { d'Ottawa: }\end{array}$ & \multicolumn{2}{|l|}{ Martin: } & $\begin{array}{l}\text { Manuscrit } \\
\text { d'Ottawa: }\end{array}$ \\
\hline $\begin{array}{l}\text { Lettres } \\
\text { historiques: }\end{array}$ & $\begin{array}{l}67 \\
69 \\
71 \\
72 \\
74 \\
75\end{array}$ & $\begin{array}{l}\mathbf{4 9} \\
\mathbf{5 0} \\
\mathbf{5 1} \\
\mathbf{5 2} \\
\mathbf{5 3} \\
\mathbf{5 4}\end{array}$ & $\begin{array}{l}\text { Lettres } \\
\text { historiques: }\end{array}$ & $\begin{array}{l}81 \\
82 \\
83 \\
84 \\
86 \\
88\end{array}$ & $\begin{array}{l}59 \\
60 \\
61 \\
62 \\
63 \\
64\end{array}$ \\
\hline & $\begin{array}{l}76 \\
78 \\
79 \\
80\end{array}$ & $\begin{array}{l}55 \\
56 \\
57 \\
58\end{array}$ & & $\begin{array}{l}46 \\
47\end{array}$ & $\begin{array}{l}\text { Relation sur } \\
\text { Marie de } \\
\text { Saint-Joseph }\end{array}$ \\
\hline
\end{tabular}

Ainsi, la disposition du manuscrit d'Ottawa est substantiellement identique à celle de Dom Claude Martin ${ }^{6}$. Le copiste suit le même ordre en éliminant, pour des raisons qui nous échappent encore, un certain nombre de lettres, et en reportant à la fin de sa collection la notice biographique consacrée à la Mère Marie de Saint-Joseph, avec la lettre qui l'accompagnait. On aura remarqué que le manuscrit d'Ottawa s'accorde avec Dom Claude Martin pour la répartition des lettres en lettres spirituelles et lettres historiques.

A priori, la dépendance du manuscrit d'Ottawa par rapport à la collection de Dom Claude est plus vraisemblable que l'hypothèse inverse. Il n'est pas probable que Dom Claude Martin, auteur d'une première collection, ait ensuite repris sa numérotation pour y insérer de nouvelles lettres découvertes dans l'intervalle (pratiquement entre 1677, date où il écrit la Vie de sa mère, morte cinq ans plus tôt, et 1681 , date où il publie sa correspondance); les erreurs de numérotation du copiste sont elles-mêmes révélatrices: les deux numéros 93 et les deux numéros 114 ; un copiste était plus à même de retrancher de la collection de Dom Claude Martin un certain nombre de lettres qui lui semblaient moins intéressantes, en donnant au fur et à mesure à la collection nouvelle une numérotation autonome, quitte à se tromper en l'établissant.

${ }^{6}$ Le manuscrit d'Ottawa ne contient pas de no 3 et 4 ; par suite d'une erreur de numérotation, deux lettres portent le $n^{\circ} 93$ (la première correspond à la lettre spirituelle 103 de Dom Claude, la seconde à la lettre 104, l'A. s'en est aperçu et est passé directement au no 95); il y a également deux lettres 114 mais cette fois, le copiste n'a pas sauté de no pour rétablir l'ordre normal. 
Mais ce sera surtout la comparaison de deux textes qui pourra nous révéler quelle est la plus ancienne des deux collections et quelle est celle qui reproduit l'autre en la corrigeant.

A la suite de Griselle, Dom Jamet avait montré l'importance des remaniements opérés par Dom Claude Martin dans la Correspondance de sa mère, en comparant le texte imprimé de 1681 , avec la longue lettre autographe de Marie de l'Incarnation à son fils, en date du 4 septembre 1641, conservée à la Bibliothèque Mazarine, à Paris ${ }^{7}$. La démonstration pouvait être faite, d'une manière presque aussi convaincante, en comparant le texte imprimé de 1681 avec la copie de quatre lettres de l'année 1644, prise sur l'original à une date indéterminée, mais sans doute assez voisine de $1644^{8}$.

Avec la lettre du 4 septembre 1641, Dom Claude Martin a fait deux lettres, une lettre spirituelle et une lettre historique; en outre, il a inventé une date pour le second des deux fragments. Les copies des lettres de 1644 permettent également de saisir en quoi a consisté le travail de remaniement effectué par Dom Claude.

Or le manuscrit d'Ottawa transforme de la même manière la lettre du 4 septembre 1641 et en fait deux lettres autonomes; pour tout le groupe des cinq lettres la version donnée par Dom Claude Martin est plus proche de l'original que celle du manuscrit d'Ottawa; aucune des variantes propres à Ottawa ne provient de l'original; ce sont des gloses ou des variantes sans aucune valeur pour l'établissement d'un texte critique; en outre, un certain nombre d'expressions dont l'éditeur de 1681 est responsable, et seul responsable, se retrouvent dans le manuscrit d'Otta-

7 Paris, Bibl. Mazarine, ms. 2467 fo 357-360; cf. E. Griselle dans Etudes (5 juin 1906), 581 ss.; id., La Vénérable Mère Marie de l'Incarnation, première supérieure des Ursulines de Québec, Supplément à sa Correspondance (Paris, 1909), 36 ss.; A. Jamet, Ecrits spirituels et historiques, II. La Correspondance de Marie de l'Incarnation (3 vol., Paris-Québec, 1935), III : 25-26, 223-234; malheureusement, dans son édition de la lettre, Dom Jamet a rajeuni l'orthographe.

8 Paris, Bibl. Nat. ms. fr. 19.961, fo 98-108; c'est à tort que Dom Jamet croyait reconnaître dans l'écriture la main de Dom Claude Martin; A. Jamet, III : 25-26, 344-353, 366-385, 385-394, 403-405. 
wa et nous font voir clairement que ce dernier s'inspire de Dom Claude.

\section{Lettre du 4 septembre 1641 :}

Mazarine, ms. 2467, fo Martin, Let. spir. 20 30-33;

\section{A. JAMET, Ecrits, III,} 223 ss.

iay esté toute cette année dans de grandes croix

pour vous

vous aves esté abandonné de vre mère et de vos parans,

cet abandon ne vous a-t-il pas été

utile?

il est vray ce que vous dites mon très cher fils iay trouvé en Canada croix

à votre occasion vos parens, a-t-il pas été avantageux?
J'ay été toute cette année en de grandes

Vous avez été abandonné de votre mère et de

cet abandon ne vous

Pour moy, mon très cher fils, ce que vous dites est véritable, que j'ay trouvé en Canada

Lettre du 2 août 1644:

B.N. ms. fr. 19. 661, fo 98-100

A. JAMET, Ecrits, III, $344 \mathrm{~s}$.

il faut une autre vocation que l'inclination naturelle

\section{Vous}

voyez que Dieu attire à son service la plus part de nos parans
Martin, Let. spir. 28

il faut une autre vocation que celle de l'inclination de la nature et du sang

Ainsi Dieu appelle la plus part de nos parents et alliez

\section{Lettre du 15 septembre 1644:}

B.N. . . . . f fo 107-108

A. JAMET, Ecrits, III, 403ss.

Je vous dis tout cecy mon très cher fils afin que vous honoriez
Martin, Let. spir. 35

$\mathrm{Je}$ vous dis tout cecy mon très cher fils afin que vous honoriez
Manuscrit d'Ottawa, Let. spir. 17

J'ai été pendant toute cette année dans de grandes inquiétudes, je veux dire de souffrances,

à votre occasion

vous avez été abandoné de votre mère et de tous vos parens, cela est vrai, mais vous voyez que cet abandon ne vous a point été désavantageux.

Pour moi, mon très cher fils, ce que vous dites est véritable, j'ai trouvé au Canada

Manuscrit d'Ottawa, Let. spir. 23

il faut pour cela une autre vocation que celle du sang et de la nature

C'est donc ainsi que dans sa grande bonté Dieu appelle nos parents et alliez

Manuscrit d'Ottawa, Let. spir. 28

Je vous dis tout ceci mon très cher fils afin que vous fassiez une 
ce bon gentilhomme

et pour vous

faire voir qu'il y a de bonnes âmes en Canada.
Monsieurde Repantigny c'est ainsi qu'il se nomme, et pour vous faire voir qu'il y a de bonnes âmes en Canada. honorable réception à Mr de Repantigny

(c'est ainsi qu'il s'appelle) et aussi pour vous dire qu'il y a de bonnes âmes au Canada.

Ces comparaisons sont absolument décisives; elles suffiraient à elles seules; aucune addition, aucune variante du manuscrit d'Ottawa ne provient de l'original. Les gloses sont à mettre uniquement au compte du copiste.

D'autres indications viennent s'ajouter à ces preuves. $L a$ Préface du manuscrit d'Ottawa s'inspire de l'Avertissement de Dom Claude; elle ne présente aucune originalité; les idées sont empruntées à l'éditeur de 1681: mention des lettres écrites à Jean de Bernières qui "roulaient" toutes sur la spiritualité et l'oraison, et perdues dès 1681 ; le nombre considérable de lettres écrites par Marie de l'Incarnation, "trois fois autant qu'on en voit rassemblées dans ce recueil"; la division de la matière en lettres spirituelles et en lettres historiques; le dernier paragraphe enfin sur la vie mystique de la grande Ursuline.

Le manuscrit d'Ottawa omet habituellement les dates des lettres; lorsqu'il les conserve, il commet les mêmes fautes de chronologie que Dom Claude ${ }^{9}$.

Les corrections du manuscrit d'Ottawa sont elles aussi révélatrices; il lui arrive fréquemment de reproduire le terme utilisé par Dom Claude Martin dans son édition, puis de le corriger et de donner un synonyme qui lui paraît meilleur; ces corrections ne sont pas le résultat d'une collation de texte, elles sont de pure fantaisie, comme celles que la comparaison établie plus haut a permis de déceler. Ainsi, dans la lettre spirituelle I de Dom Claude (Elévation du manuscrit d'Ottawa), là où l'édition de 1681 mettait: "vous leur ôtez", le manuscrit met: "vous (leur) ôtez à ces personnes", leur ayant été biffé au cours de la rédaction; le copiste a jugé que le terme n'était pas suffisam-

${ }^{9}$ Les lettres, surtout celles des premières années, ne se trouvent pas toujours dans l'ordre chronologique; Dom Jamet a tenté de corriger les dates chaque fois que la chose lui semblait possible. 
ment clair et a tenu à le préciser. Les exemples analogues pourraient être aisément multipliés. Un peu plus loin, dans la même lettre, ayant d'abord transcrit conformément à l'édition de Dom Claude: "je demeure pour jamais collée à mon grand Amour", il biffe le mot "collée" et le remplace par "étroitement unie"; il a donc certainement sous les yeux l'édition de 1681 qu'il recopie; il ne peut aucunement s'agir d'une collation de texte, car les gloses ajoutées par l'auteur du manuscrit d'Ottawa s'insèrent dans le texte et ont été écrites dès la première rédaction.

Enfin, il arrive assez fréquemment que le copiste intègre les notes marginales de l'édition de 1681 dans le texte même des lettres ou dans les titres qu'il leur donne ${ }^{10}$, ou bien il les reproduit en post-scriptum ${ }^{11}$.

Insister sur ces preuves complémentaires serait superflu; elles ont surtout valeur d'appoint, la démonstration ayant été amplement réalisée par la triple confrontation de l'original (ou des copies prises sur l'original) avec l'édition de 1681 et le manuscrit d'Ottawa, pour les quelques parties de la Correspondance où une telle confrontation est possible.

De la sorte, lorsque l'auteur du manuscrit d'Ottawa parle de l'original ("Il est dit dans l'original", Pour servir de Préface, p. 2), il se réfère uniquement à l'édition imprimée de 1681 .

Quelle fut donc l'intention du copiste qui s'appliqua à retranscrire, en abrégeant, en corrigeant et parfois en glosant de façon notable, la collection de Dom Claude Martin ? Il s'en explique en son Introduction qu'il a intitulée: "Pour servir de Préface":

Ce seroit vraiment dommage qu'un bien si précieux se perdit faute de réimpression, c'est ce qui arrivera cependant par suite de l'indifférence qu'on a pour certains ouvrages de piété; on ne les trouvera plus que dans les Bibliothèques des grandes villes où ils

10 Cf. par exemple Let. spir. 27 (34 de Dom Claude Martin).

11 Par exemple Let. spir. I (3 de Dom Claude). 
ne seront gueres plus connus, et lus pas davantage. Cependant je ne crains pas de dire qu'elles me semblent être dignes d'être mises à côté de celles de St François de Sales et de Fenelon pour l'onction, la délicatesse, et la beauté du stile.

L'auteur se proposait donc de livrer sa propre collection à l'impression, parce que l'édition de 1681 ne se trouvait plus que dans les bibliothèques des grandes villes, ou presque.

Dès lors, il serait très intéressant de déterminer le lieu et la date de composition du manuscrit, car nous aurions par le fait même déterminé le lieu et la date de cette "réimpression" avortée. Malheureusement, les arguments de critique interne et externe ne permettent pas d'apporter toutes les précisions souhaitables; du moins, est-il possible d'arriver à une approximation suffisante.

Le lieu tout d'abord. Deux hypothèses peuvent être envisagées: ou bien le manuscrit est originaire du Canada et l'auteur de la collection avait l'intention de faire paraître l'ouvrage au Canada pour les communautés d'Ursulines du nouveau monde, ou bien le manuscrit est français, composé en France, à l'intention des prêtres, religieuses et chrétiens cultivés adonnés aux lectures spirituelles.

Le seul examen de la Préface apporte des lumières, à la fois sur le lieu et la date; le copiste dit en effet en substance: on ne trouvera bientôt plus les lettres de Marie de l'Incarnation que dans les bibliothèques des grandes villes; il pense aux bibliothèques publiques, cela est clair; or, ni au XVIII ${ }^{\mathrm{e}}$ siècle, ni dans la première moitié du XIX ${ }^{e}$ siècle, un Canadien n'aurait pu parler de "grandes villes" ni de "bibliothèques" publiques, les seules localités importantes étant Québec, Montréal et TroisRivières; par ailleurs, le copiste a déclaré un peu auparavant son intention de livrer les lettres de l'Ursuline à la "réimpression". "Ce seroit vraiment dommage qu'un bien si précieux se perdit faute de réimpression." On sait que les premières imprimeries canadiennes furent créées un peu avant 1767 par Brown et Gilmore et en $\mathbf{1 7 7 4}$ par Fleury Mesplet et que la période des 
"incunables canadiens" s'étend sur la fin du XVIII et au moins le premier tiers du XIX $\mathrm{XI}^{\mathrm{e}}$ siècle; il n'est donc pas possible de considérer le copiste comme un Canadien composant pour le Canada un recueil de lettres de Marie de l'Incarnation; d'autres raisons viendront corroborer celle-ci; on les trouvera plus loin.

Mais dès maintenant, il convient de remarquer qu'un Français ne pouvait parler des "bibliothèques des grandes villes" avant 1792, pour la bonne raison qu'il n'existait pas auparavant de véritables bibliothèques publiques. Les "bibliothèques des grandes villes" (c'est-à-dire les bibliothèques publiques des préfectures et, parfois, des sous-préfectures) ont été créées pour recueillir les fonds des bibliothèques conventuelles après l'abolition des ordres religieux par l'Assemblée Constituante (1791). Comme d'autre part, il est certain qu'une œuvre de cette envergure n'a pu être entreprise avant le Concordat de 1802, force est bien de donner à la collection une date postérieure; d'autres indices peuvent être relevés en faveur d'une date assez basse, postérieure à la Révolution française.

Nous avons dit plus haut que l'auteur n'était pas canadien; l'argument présenté pouvait suffire; il en est d'autres:

- La traduction d'Onontio (écrit dans le manuscrit "Onuntio") est fautive; les indiens se sont servis de la traduction littérale de Montmagny (Mons magnus) en leur langue pour désigner le gouverneur français, Charles Huault de Montmagny ayant été le premier gouverneur et lieutenant-général de la Nouvelle France, de 1636 à $1648^{12}$. Or dans sa lettre historique 29 (32 de Dom Claude), l'auteur glose: "Onuntio (qui signifie Maître débonnaire !)" ${ }^{13}$.

- Il est incapable de lire le son 8 (ou), couramment employé dans la transcription des noms indiens par Dom Claude Martin; ainsi dans la lettre spirituelle 82 (90 de Dom Claude) Outa8ak est remplacé par Out...; dans la lettre spirituelle (21 de Dom

12 Cf. art. de Jean Hamelin dans Dictionnaire biographique du Canada (Presses de l'Université Laval, 1966), 383.

13 Ibid., 316. 
Claude) Pigar8ich est purement et simplement omis; de même Ani8gan dans la lettre historique 29 (32 de Dom Claude) ${ }^{14}$.

- Mieux encore, la lettre historique 32 de Dom Claude parlait du "Commandant des Trois-Rivières"; l'auteur du manuscrit écrit "commandant le lieu dit les Rivières"; cela serait inconcevable de la part d'un Canadien.

La date, avons-nous dit, est certainement postérieure à la Révolution française; la forme de l'écriture et l'orthographe pourraient appartenir à la fin du XVIII ${ }^{e}$ siècle, ce qui n'infirme nullement la conclusion avancée plus haut, car un auteur travaillant au début du $\mathrm{XIX}^{\mathrm{e}}$ siècle à la réimpression d'un ouvrage de spiritualité peut être, par toute sa formation, un homme du $\mathrm{XVIII}{ }^{\mathrm{e}}$ siècle et avoir gardé ses habitudes littéraires. L'examen du papier et de son filigrane a conduit les spécialistes à y voir un papier du XVIII ${ }^{\mathrm{e}}$ siècle, et plutôt du XVIII ${ }^{\mathrm{e}}$ siècle finissant ${ }^{15}$.

L'auteur de la collection écrit en France, avons-nous dit; il ne peut être antérieur à la Révolution. Car il ignore plusieurs choses élémentaires touchant la Congrégation bénédictine de

${ }^{14}$ Les exemples pourraient aisément être multipliés, soit que l'auteur supprime un nom sauvage en le remplaçant par son initiale, comme Tokhiahenchiaron, remplacé par $T$. dans la Lettre historique 29 (32 de Dom Claude), soit qu'il interprète à sa manière un trait de mœurs; ainsi Dom Claude écrivait dans la lettre historique 32 (p. 395): "Après avoir pétuné à la façon des sauvages et mangé quelques pruneaux", le copiste interprète (let. hist. 29, p. 316) : "Après avoir pris quelques rafraîchissements"; de même let. hist. 84, p. 648: "Ils entourèrent (un enfant nouvellement baptisé) de peaux et de porcelaines", devient dans le manuscrit d'Ottawa: "Ils l'entourèrent de peaux et le décorèrent de joujoux de porcelaine"; le copiste ignore donc ce que sont les wampums; les voyages en canot deviennent chez lui des randonnées en voiture: "Il y avoit surtout une jeune veuve qu'on menoit exprès pour donner bon exemple aux femmes hiroquoises. Elle fut prise captive par un barbare qui luy voulut ravir son honneur à une décente qu'on fit à terre" (Let. hist. 56, p. 538); le manuscrit d'Ottawa porte: "Cette jeune femme donc fut faite captive par un barbare qui voulut abuser d'elle au moment d'une dessente de voiture" (Let. hist. 42, 345).

15 Pour M. Thomas, de la Bibliothèque Nationale de Paris, les filigranes du papier appartiendraient à un type rencontré dans les papiers anglais

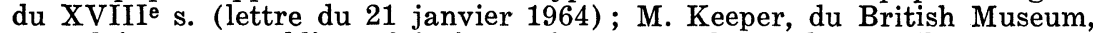
consulté sur ce problème, faisait savoir par une lettre du 8 juillet 1964 que les filigranes étaient inconnus des répertoires; M. Pennington de McGill University Library de Montréal pensait qu'il pouvait s'agir d'un papier français des environs de 1800 , en tout cas certainement pas d'un papier américain (lettre du 26 janvier 1965); le Père L. Campeau s.j. était du même avis (lettre du 16 février 1965). 
Saint-Maur qu'il nomme une "Compagnie" (Lettre spirituelle 101, p. $222=111$ de Dom Claude, p. 254). Dans la lettre du 12 octobre 1668 qui mentionne l'élévation de Dom Claude à la fonction d'Assistant général de la Congrégation, il introduit une parenthèse: "Il paroît que c'étoit celle de Secrétaire ou de Grand Vicaire". Pareille méconnaissance est difficile à concevoir de la part d'un ecclésiastique, en France, avant 1791, car chaque diocèse possédait au moins deux, souvent trois, parfois sept ou huit maisons de "mauristes"; par contre elle est tout à fait vraisemblable après la Révolution et le Concordat de 1802.

Tous ces indices viennent appuyer notre premier élément de datation: l'argument tiré des "bibliothèques de grandes villes" qui, seul, pourrait être considéré comme suffisant.

Il reste à formuler une hypothèse sur le rédacteur du manuscrit d'Ottawa. Etant donné ce que nous avons sur la date approximative de l'œuvre, il semblerait logique de voir en lui un ecclésiastique, voire un aumônier d'Ursulines, qui voulait donner à l'Ordre en voie de réorganisation après la période révolutionnaire une nouvelle édition des lettres de Marie de l'Incarnation. Ce ne peut être, pour le moment, qu'une hypothèse de travail; un indice cependant la rend plus vraisemblable encore: dans les lettres spirituelles 55, p. 133, et 56, p. 134 (63, pp. 140-142, et 64, pp. 143-144 de l'édition de Dom Claude), l'auteur du manuscrit d'Ottawa omet tout ce qui vise les difficultés surgies, au couvent de Québec, entre religieuses venues de la Congrégation de Bordeaux et celles qui avaient précédemment appartenu à la Congrégation de Paris; ce sont des souvenirs qu'il n'était pas utile de rappeler, si le livre devait être lu principalement par des Ursulines.

La comparaison du manuscrit d'Ottawa avec l'édition anonyme, aujourd'hui presque introuvable, de 1857 (Clermont-Ferrand) ${ }^{16}$ montre qu'il n'y a aucune parenté entre les deux, sinon par l'intermédiaire de Dom Claude Martin. L'édition de Cler-

16 Cette édition est malheureusement peu accessible; il en existe un exemplaire à la Bibliothèque Nationale de Paris, sous la cote $8^{\circ} \mathrm{Ld} 1723$, les Ursulines de Québec en possèdent un exemplaire. 
mont-Ferrand ne se présente pas comme une édition complète, mais comme un simple choix de lettres; son volume est à peu près équivalent à celui du manuscrit d'Ottawa; car l'édition imprimé compte 388 pages, et le manuscrit 415 . Mais le contenu diffère: l'édition de Clermont se limite aux seules lettres historiques, tandis que le manuscrit d'Ottawa donne la primeur aux lettres spirituelles.

Quoi qu'il en soit, la valeur du manuscrit d'Ottawa est nulle pour une édition critique des lettres de Marie de l'Incarnation, car il ne présente rien qui ne soit issu de l'édition imprimée de 1681 ou de l'imagination du copiste; il constitue seulement un chaînon qui nous permet de constater l'intérêt permanent suscité par la Correspondance de la grande Ursuline, puisqu'au lendemain des bouleversements révolutionnaires, il s'est trouvé quelqu'un pour préparer une réédition rajeunie du recueil publié naguère par Dom Claude Martin.

DOM GUY OURY, Abbaye de Solesmes, France.

\section{LA CONSULTATION DES ARCHIVES SECRETTES DU VATICAN}

L'Observatore Romano du 8 décembre 1966 publie la note ci-après de Mgr Martino GIUSTI, préfet des archives secrètes du Vatican, déclarant ouvertes à la consultation publique les archives secrètes jusqu'à la fin du pontificat de Pie IX:

[...] Il y a eu cette année quatre-vingt-six ans que Léon XIII, en 1880 , décida d'ouvrir pour la première fois les archives secrètes du Vatican à la consultation publique.

Parmi toutes les archives historiques, celles-ci revêtent une importance et un intérêt universels en raison des documents intéressant aussi bien l'histoire de la civilisation chrétienne en général que l'histoire de chaque nation. Aussi, à partir de ce moment, d'innombrables historiens sont-ils venus de tous les pays pour faire des recherches dans les différents domaines des sciences historiques.

Léon XIII avait établi comme limite pour les recherches l'année du Congrès de Vienne (1815). Cette limite fut ensuite graduellement étendue. Pie XI l'avait finalement fixée à 1846 , c'est-à-dire à la fin du pontificat de Grégoire XVI.

Aujourd'hui, S. S. Paul VI a décidé qu'à partir du début de l'année qui vient (1 $1^{\text {er }}$ janvier 1967$)$, les limites de la consultation seraient étendues, et que seraient accessibles les archives du pontificat de Pie IX (16 juin 1846-7 février 1878).

On sait que cette période a connu des événements historiques particulièrement importants, non seulement du point de vue religieux et ecclésiastique, mais aussi du point de vue politique. Qu'il suffise de citer: le premier Concile du Vatican (1869-1870), la fin du pouvoir temporel des papes et des Etats pontificaux (20 septembre 1870). D'autre part, le long pontificat de Pie IX offre aux historiens un riche matériel de recherches. Rappelons, par exemple, les précieuses correspondances de la Secrétairerie d'Etat, les archives des nonciatures, le fond et les archives de Pie IX, les archives du premier Concile du Vatican, les lettres des importants dicastères et services de la Curie romaine relatifs à l'administration des Etats pontificaux, et surtout au gouvernement de l'Eglise universelle. (La Documentation catholique, \# 1486, 15 janvier 1967). 